\title{
¿Hubo infectólogos en la Antigua Roma?
}

\author{
Walter Ledermann D.
}

\section{Were there infections diseases specialists in the Ancient Rome?}

The existence of infectious diseases specialists in Ancient Rome is unlikely, but there were at least three authors able of keen observations on infectious matters, with enough merit to be considered our predecessors: Varro, Columella and Vitruvius, none of them physicians. Varro, in his first Book on Agriculture recommended "build the houses distant from swamps, because certain minute creatures are bred which cannot be followed with the eyes but which float in the air and enter the body through the mouth and nose, giving rise to severe diseases". Also in a text of agriculture and in the same sense, Columella says "that with heat a swamp releases a pestilential vapor and produces a very dense swarm of insects, which come flying over us armed with harmful stings ...". Vitruvius, the great architect, was worried about drinkable water: its sources and properties, how to obtain it and the methods for testing its quality. The concern on its distribution and disposal of sewage started on 614 B.C., little after the foundation of Rome, with the building of the first aqueduct, the Aqua Marcia. This aqueduct in Trajan's times (century II A.D.), reached a total of $443 \mathrm{~km}$, with 49,500 meters of arcades, which were up to 32 meters high, plus $2.4 \mathrm{Km}$. of an underground net. This system released $947.200 \mathrm{~m}^{3}$ of water per day, two thirds of which were for public use and one third for private customers.

Key words: Infectious diseases specialists, Ancient Rome, Varro, Columella, Vitruvius.

Palabras clave: Infectólogos, Antigua Roma, Varrón, Columela, Vitruvio.
Recibido: 5 de enero de 2010 Aceptado: 16 de enero de 2010

Correspondencia a: Walter Ledermann Dehnhardt oncemayor@gmail.com
S $i$ atendemos literalmente al término, con seguridad no. La palabra castellana infecto es el participio irregular del verbo inficiar (sin: inficionar); tomado como adjetivo, significa inficionado, contagiado, pestilente, corrompido; de estas acepciones, la más difundida en el país es pestilente. Al crearse en Chile hace 25 años la Sociedad que nos agrupa, se eligió infecto, como lógica consecuencia del colonialismo cultural norteamericano, naturalmente aceptado, que aflige a los hispánicos, y quienes hasta entonces vivíamos felices cual especialistas en enfermedades infecciosas, adoptamos el incómodo nombre de infectólogos, pasando a ser estudiosos de lo pestilente o, peor aún, estudiosos que apestan. En el latín de Roma, infección era infectio: bien pudimos ser infectiólogos, que sonaría más elegante a oídos de nuestros pacientes, o, partiendo la palabra castellana infección, infecciólogos. Y mayor prestigio gozaríamos si hubiésemos, como nuestros amigos microbiólogos y hematólogos, elegido una raíz griega, más rancia todavía que una latina. Pero, en fin, el daño ya está hecho y debemos sobrellevar el mote.

Ahora bien, si atendemos al significado intrínseco de la palabra, como la entendemos nosotros en el presente, mal pudo haber infectólogos en la Antigua Roma. Hubo, en cambio, intelectuales capaces de agudas observaciones en materia de infecciones, con merecimientos suficientes para postularse como nuestros predecesores. Se nos vienen de inmediato a la mente los nombres de Varrón,
Columela y Vitruvio, ninguno de ellos médico, cosa que poco importa, dado que en aquella época el conocimiento que sobre las enfermedades, infecciosas o no, tenían los así llamados médicos no era muy superior al de cualquier ciudadano.

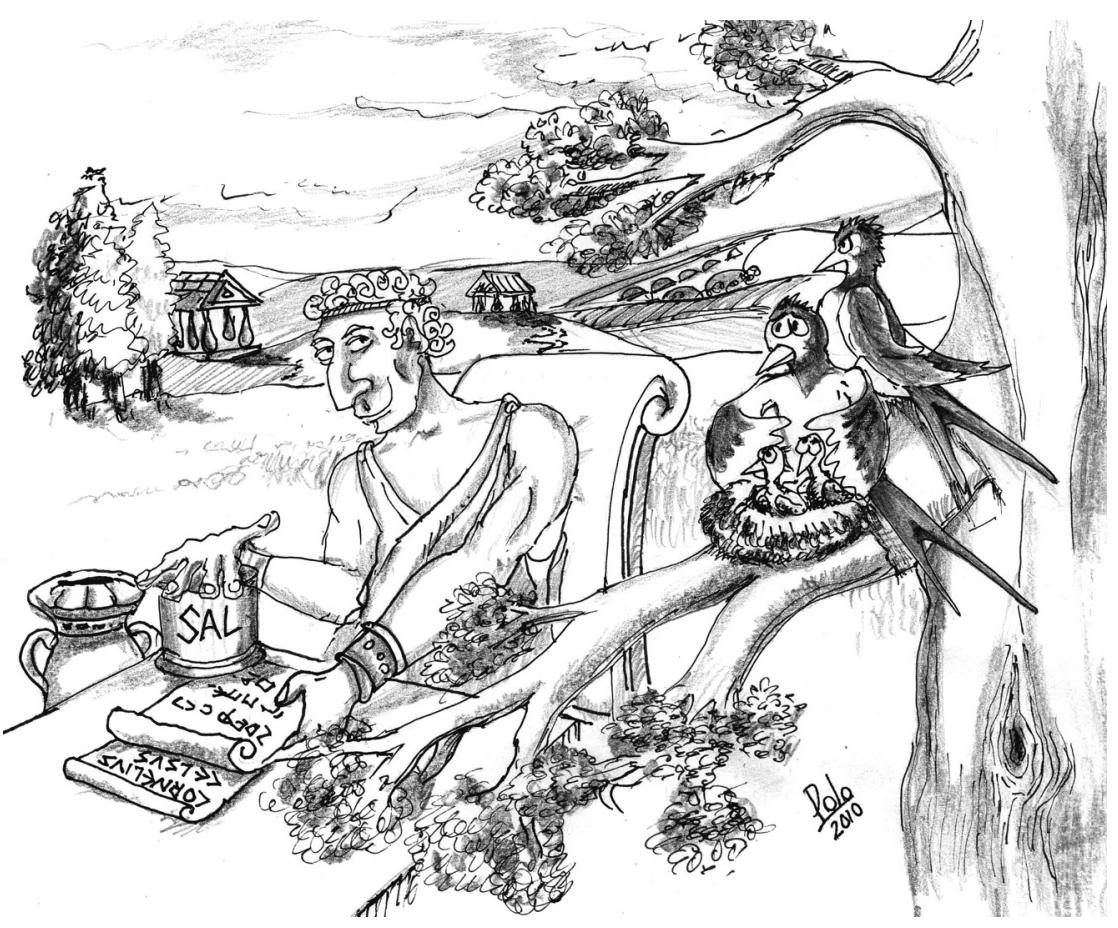


La cultura médica romana era heredera de la griega, y ésta, a su vez, debía más de algo a las babilónica, egipcia y hebrea, a las cuales por ahora no tocaremos. Su máximo exponente, después de Galeno, era Cornelio Celso, el autor de la célebre De Re Medica, aunque algunos historiadores dudan que alguna vez hubiese ejercido la medicina, bastando para dudas esta jocosa recomendación tomada de su libro: Oigo a cada paso decir que quien come una cría de golondrinas se verá todo el año libre de anginas; también se pretende que se obtiene este resultado conservando la cría en sal y cuando se presenta la enfermedad se la tuesta, se la machaca para hacerla polvo, se la disuelve en agua y se la da a beber al enfermo... Esta opinión popular, por tener a su favor la autoridad de gentes dignas de fe, y porque por lo demás no ofrece peligro alguno, he creído que debía darle acogida en mi trabajo, aunque no la he leído en los escritos de los médicos... ${ }^{1}$. Nótese del texto como este médico, con nula seriedad científica, habla "de oídas": sus palabras no son fruto de la observación ni del estudio o siquiera de la experiencia propia o de sus colegas, sino, en suma, un "cuento de viejas", como diríamos hoy día; adviértase, además, la enormidad del disparate expuesto, indigno de cualquier sujeto que se repute médico. Más serios nos parecen los conceptos de los legos Varrón, Vitruvio y Columela; vamos, pues, a ellos.

Marco Terencio Varrón vivió en la Roma anterior a Jesucristo. Era natural de Reate, hoy conocida como Rieti, en tierra sabina, en el año 116 A.C, y vivió hasta el año 27 del siglo siguiente. Era hombre múltiple y cuesta definir su actividad principal, soliendo citársele como escritor, filólogo y polígrafo. Como filólogo era discípulo de Estilón; como filósofo, se formó a la vera de Antíoco de Ascalón. Sin embargo, fue famoso por sus hazañas militares, llegando a ser nada menos que el lugarteniente de Pompeyo, siendo condecorado por su valor durante la lucha que éste emprendiera contra los piratas.

Aunque escogió el bando equivocado y luchó por Pompeyo en Lusitania, César, el ilustre vencedor, no le tomó ojeriza y le encargó la dirección de las bibliotecas públicas. Pero, ¡ay!, los tiempos eran difíciles y el poder iba de mano en mano: César fue asesinado al poco tiempo, Varrón perdió el cargo, sus bienes fueron confiscados por Marco Antonio, quien de paso quemó su biblioteca, pues es sabido que las armas odian a los libros, y lo condenó a muerte.

Sin embargo, debe haber sido hombre muy notable-era amigo de Cicerón y Plutarco decía de él que era el mayor lector de su época- pues pronto ganó el favor de Octavio, quien le dio una jugosa indemnización y lo volvió a sus bibliotecas. Entonces tuvo tiempo para escribir sobre filosofía, agricultura y varios temas más; sumando poesía y teatro, se llega a unas 600 obras (si hemos de creer a
Wikipedia, que le atribuye también la invención de la mortadela). Poco se conserva de tamaña obra, apenas 74 y en su mayor parte fragmentos, pero ahí está lo más valioso para la infectología: Rerum rusticarum o "Los tres libros de agricultura", y el trozo sobreviviente de Disciplinarum libri $I X^{2}$.

En el primero de sus libros de agricultura hace referencia a las causas del paludismo, que ya era endémico en Roma y en toda Italia. Samuel Fienkelman ${ }^{3}$, ha traducido de una edición inglesa el texto en que Varrón recomienda la mejor ubicación para una casa de campo, al pie de una colina arbolada, con amplios pastizales, de manera de exponerla a los vientos más favorables que soplen en la región. Y, más adelante, una advertencia que remece toda la historia de la microbiología: también deben tomarse precauciones en la vecindad de los pantanos, tanto por las razones dadas como porque allí crecen ciertos animales, tan diminutos que no se pueden seguir con los ojos y flotan en el aire y entran al cuerpo por la boca y la nariz causando graves enfermedades. Pero nuestro arcaico infectólogo no se detiene ahí y entra de lleno a recomendar una profilaxis con luz ultravioleta para evitar la infección: al estar (la casa) expuesta al sol durante todo el día resulta más saludable, ya que cualquier animálculo que se haya criado cerca y entre a la casa es alejado por el viento o muere rápidamente por falta de humedad.

Analizando la historia de malaria en Italia, Snowden atribuye a Varrón la idea que un insecto pudiera tener un rol activo en el contagio de la malaria, para descalificarlo a continuación, diciendo que en ausencia de una prueba empírica válida y de una adecuada comprensión del mecanismo involucrado, (esta idea) no era relevante, $n i$ convincente ni mucho menos útil ${ }^{4}$. Como Varrón creyó que los animálculos penetraban al hombre vía oral, sus aportes infectológicos quedan, a nuestro juicio, reducidos a dos: - Existirían en las zonas pantanosas animálculos, seres invisibles a simple vista, capaces de transmitir enfermedades (hoy: microbios).

- Estos animálculos requerirían de la humedad para medrar, de manera que una exposición continuada al sol sería capaz de eliminarlos (hoy: profilaxis, saneamiento, LUV).

En cuanto a la mortadela, si no la inventó, al menos fue el primero en usar el término. Su importancia para la infectología es nula, dado que los infectólogos estamos tan inmersos en nuestra especialidad, que los embutidos sólo nos importan en relación a la posibilidad de albergar toxina botulínica, en tanto que el colesterol y el síndrome metabólico nos dejan fríos.

Sería Columela, medio siglo después de Varrón, quien relacionara la presencia de los mosquitos en las zonas pantanosas con la aparición de las fiebres, es decir, con el paludismo, como se viene repitiendo sin cesar a 
consecuencia de un párrafo que escribiera a mediados del siglo I de nuestra era.

Lucio Junio Moderato Columela vivió en el siglo I D.C. y es posterior a Varrón, aunque la fecha exacta de su nacimiento en Cádiz se desconoce. Hoy en día se le estimaría español, pero en su época fue ciudadano romano, al igual que su compatriota Séneca, al cual conoció y frecuentó en Roma. Experto en agricultura, dio a conocer sus conocimientos en la obra De re rustica, donde trata de los trabajos del campo, y que suele editarse como "Los doce libros de la Agricultura"5.

Fantini afirma que el mosquito siempre ha estado ligado a la malaria y que en los textos del Ochocientos, es decir, el siglo XIX, "se hace constante referencia a algún pasaje de Columela, que habla del paludismo que producen venenos y animales infectados dotados de aguijones". El texto de Columela aparece en el capítulo V del primero de los doce libros de agricultura : nec paludem vicinam esse oportet aedifici nec iuncta militarem viam quod illa caloribus noxium eructat et infestis aculeis armata gignit animalia, quae in nos densissimis examinibus involant. .

La clásica versión al castellano de Álvarez de Sotomayor $^{7}$ traduce paludem por laguna y militarem viam por camino real, pero es aceptable: Tampoco conviene que haya laguna cerca de los edificios ni camino real, porque aquélla desprende con los calores un vapor pestilencial y produce enjambres espesísimos de insectos, que vienen volando sobre nosotros armados de aguijones dañinos...

Así acotado, el texto parece convincente, pero si completamos el párrafo, no queda claro que atribuya la enfermedad palustre a la picadura de los insectos: ... y al mismo tiempo echa afuera las pestiferas serpientes de la tierra y del agua, que privados de la humedad del invierno $y$ envenenados con el lodo y el cieno fermentados, son causa muchas veces que se contraigan unas enfermedades desconocidas, cuyos motivos ni aun los médicos pueden descubrir. En suma, los insectos con aguijón son uno de los múltiples factores que causan enfermedades "desconocidas".

Lucius Junios Moderatus Columella, tal era su nombre latino, no tuvo ni remotamente una vida tan variada, agitada y brillante como Varrón, conformándose sólo con sus conocimientos agrícolas. Si embargo, al escribir los doce libros con los que pasaría a la posteridad, como si lo intuyera, intentó dejar prueba de su capacidad poética y escribió el décimo, dedicado a los jardines, en hexámetros, imitando a las Geórgicas de Virgilio, pero fracasó en su intento de rozar la altura del maestro de la poesía romana ${ }^{5}$.

Digamos, de paso y ya que estamos en él, que Virgilio en sus Geórgicas tuvo un contacto colateral con la infectología, al relatar en las mismas Geórgicas, con indescriptible belleza, la peste -¿carbunco ?- que asolara los campos itálicos, diezmando los ganados lanar y caballar, y de la cual no escaparon ni los mismos perros que los guardaban: cuando empieza a recrudecer la peste, entonces les arden los ojos; su resuello se hace profundo, dificil y entrecortado por los gemidos, dilátanse los ijares con prolongados sollozos; una sangre negra les mana de la nariz y la seca y rígida lengua les oprime las hinchadas fauces ${ }^{8}$.

Junto a Varrón y a Columela suele citarse al arquitecto Marco Vitruvio Polion, afirmándose la común convicción entre ellos de la propagación de la malaria mediante la picadura de insectos o por aguas pantanosas, y que por dicha razón se habrían realizado obras cívicas como acueductos, alcantarillado y baños públicos, para asegurar tanto el acceso a agua potable y la necesaria eliminación de excretas, es decir, todo un programa de salubridad muy adelantado para su época, que las invasiones bárbaras arruinarían por completo.

Sólo una vez en sus diez libros de arquitectura se refiere Vitruvio tangencialmente a las lagunas y a sus "efluvios venenosos". En el libro 1, capítulo IV, donde trata "de la elección de los parajes sanos", dice: En la fundación de la ciudad, será la primera diligencia la elección del paraje más sano. Lo será siendo elevado, libre de nieblas y de escarcha; no expuesto a aspectos calurosos ni frios, sino templados. Se evitará también la cercanía de lagunas, porque viniendo a la ciudad las auras matutinas al salir el sol, traerán consigo los humores nebulosos que alli nacen, juntamente con los hálitos de las sabandijas palustres esparciendo sobre el cuerpo de los habitantes sus venenosos efluvios mezclados con la niebla, haciendo pestilente aquel pueblo9.

Es posible que Columela, que no era arquitecto, al momento de escribir su texto se haya limitado a copiar a Vitruvio, toda una autoridad en arquitectura, cuyos libros fueron escritos entre los años 23 a $27 \mathrm{AC}$, pero su mérito radica en hablar específicamente de los aguijones. En cuanto a Vitruvio, otro aporte a la salubridad aparece en su octavo libro, que trata del agua potable : sus fuentes y sus cualidades, los métodos para obtenerla y los métodos para probar su calidad.

En este campo Vitruvio estaba lejos de ser un precursor, ya que la preocupación por el abastecimiento de agua y disposición de excretas había nacido casi al momento de la fundación de Roma y en el 614 A.C., bajo el reinado de Anco Marcio, uno de los monarcas latinos (o sabinos), se había construido el primer acueducto -el Aqua Marciaque llevaba a la ciudad agua desde un manantial situado a unos diez kilómetros. Los reyes etruscos continuaron la tarea y bajo Tarquinius Priscus se empezó la canalización subterránea, terminada bajo el reinado de Tarquinius Superbus, quien también hizo construir la Cloaca Máxima para llevar los desechos hacia el Tíber ${ }^{10}$.

A mayor abundamiento, Frontin, quien dejara el mando de un ejército en Bretaña para asumir como curador 
o director de aguas de Roma, cargo que ejerció desde Nerón a Trajano, en los siglos I y II D.C., cuenta que bajo el imperio de este último había ya en la ciudad nueve acueductos, comprendiendo el citado Aqua Marcia, más Appia Claudia, Aniovetus, Repula, Julia, Virgo, Alsietina, Claudia y Anio Nova, con un total de $443 \mathrm{Km}$, con 49.500 metros de arcadas de hasta 32 metros de altura, más 2,4 $\mathrm{Km}$ de red subterránea. Éstos entregaban $947.200 \mathrm{~m}^{3}$ por día, de los cuales dos tercios eran para uso público y un tercio privado, lo que hacía $2,7 \mathrm{~m}^{3}$ por persona y por día. Gran parte del agua se perdía en el camino, por fisuras y filtraciones; el resto se almacenaba en la ciudad en 247 depósitos, desde los cuales se distribuía por tubería de plomo y de bronce. Concesiones de agua se obtenían como favores del emperador y relata Frontin que de una sola derivación se habían hecho 13.594 concesiones a particulares.

En cuanto a la disposición de excretas, mucho antes del Imperio las casas ya tenían letrinas, instaladas en la vecindad de las cocinas; las letrinas públicas fueron muy posteriores y se establecieron recién en tiempos de Diocleciano, a fines del siglo III DC ${ }^{11}$.

Volviendo a Vitruvio, es también conocido como inventor e ingeniero, por el diseño de una rueda hidráulica muy eficaz para moler granos, pero más aún como anatomista, porque Leonardo da Vinci habría seguido sus indicaciones sobre las proporciones del cuerpo humano en su famoso dibujo "El hombre de Vitruvio" que está en la Galería de la Academia, en Venecia ${ }^{12}$

Escribe Leonardo, al pie de su dibujo: Vitruvio, el arquitecto, explica en su obra sobre arquitectura que la naturaleza dispone las medidas del cuerpo humano de la siguiente manera: 4 dedos forman 1 palma, 4 palmas son 1 pie, 6 palmas son 1 codo y 4 codos la altura de un hombre. Y estas eran las medidas que usaba en sus edificios. Si abre las piernas de forma que su altura disminuya en 1/4 y extiende los brazos, levantándolos hasta que los dedos corazón estén a la altura de la parte superior de su cabeza, el centro de las extremidades estará en el ombligo y el espacio entre las piernas formará un triángulo equilátero ${ }^{13}$.

No deja de ser curioso que Vitruvio, tan bueno para escribir libros de arquitectura, pareciera haber construido poco: la única obra proyectada y construida en su vida y sobre la cual existe certeza, sería la Basílica en la colonia Julia Fanestris (o Fanum Fortunae), fundada por Augustus alrededor del 19 D.C., en lo que hoy es la ciudad adriática de Fano, provincia de Pesaro, siendo probable que le pertenezca también el diseño de toda la colonia. Por desgracia, ésta fue destruida por los visigodos el año 540; habiéndose perdido los planos, resta sólo la descripción que el autor hace en el capítulo uno de su V
Libro de arquitectura, que comienza con la célebre frase : Non minus summun dignitatem et venustatem possuant habere comparationes Basilicarum quo genera coloniae Juliae Fanestri collocavi curavique faciendam cuius proportiones et symmetriae sic sunt constitutae... ${ }^{14}$. A partir de 1486, con la primera edición de De Arquitectura por Giovanni Sulpici, una pléyade de artistas del Renacimiento se abocó a interpretar el texto vitruviano, dando lugar a una copiosa documentación gráfica, que ha permitido en este siglo XXI a Paolo Taus, partiendo del estudio de los sitios arqueológicos y usando moderna tecnología computacional, reconstruir virtualmente no sólo la Basílica, sino la colonia entera de Fanum Fortunae ${ }^{15}$.

¿Y Galeno? Un gran maestro, sin duda; no obstante, al igual que Celso, fue más que todo un compilador y sobre materia infecciosa no registra ningún aporte personal. Quedamos limitados, entonces, a un trío de agudos observadores de la enfermedad nacional que el paludismo era para la península itálica: si bien no podemos considerarlos infectólogos, son dignos antecesores de los grandes investigadores italianos en el campo de la malaria, como Ettore Marchiafava y Battista Grassi.

\section{Resumen}

Si mal pudo haber infectólogos en la Antigua Roma, hubo al menos intelectuales capaces de agudas observaciones en materia de infecciones, con merecimientos suficientes para postularse como nuestros predecesores: Varrón, Columela y Vitruvio, ninguno de ellos médico. Varrón, en el primero de sus Libros de Agricultura, recomendaba ubicar la casa evitando los pantanos, porque alli crecen ciertos animales, tan diminutos que no se pueden seguir con los ojos y flotan en el aire y entran al cuerpo por la boca y la nariz causando graves enfermedades. También en un texto de agricultura y en el mismo sentido, Columela dice que una laguna desprende con los calores un vapor pestilencial y produce enjambres espesísimos de insectos, que vienen volando sobre nosotros armados de aguijones dañinos... Vitruvio, el gran arquitecto, se ocupó del agua potable: sus fuentes y sus cualidades, cómo obtenerla y los métodos para probar su calidad. Esta preocupación por la distribución de aguas y disposición de excretas había nacido en el 614 A.C., poco después de la fundación de Roma, con la construcción del primer acueducto, el Aqua Marcia, para alcanzar en tiempos de Trajano (siglo II D.C.) un total de $443 \mathrm{~km}$, con 49.500 metros de arcadas de hasta 32 metros de altura, más 2,4 $\mathrm{km}$ de red subterránea. Éstos entregaban $947.200 \mathrm{~m}^{3}$ por día, de los cuales dos tercios eran para uso público y un tercio privado. 


\section{Referencias}

1.- Aurelio Cornelio Celso. Los Ocho libros de la Medicina. Editorial Iberia S.A., Barcelona 1966; IV: IV. 1.

2.- McLean Harper G. Varro, Marcus Terentius. En: Collier's Encyclopedia. Crowell Collier and MacMillan, Inc, USA, 1967; 23: 45.

3.- Finkielman S. Marco Terencio Varrón y la causa de las enfermedades. Medicina (B. Aires) mayo/jun 2007; 67 (3).

4.- Snowdown F M. La conquista della malaria. Una modernizzazione italiana 1900-1962. Giulio Einaudi Editore s.p.a., Torino 2000; 47-8.

5.- MacLean Harper L. Columella, Lucius Junios Moderatus. En: Collier's Enciclopedia. Crowell Collier and MacMillan Inc.,USA 1967; 7: 39.

6.- Fantini B. Uno zoologo e la malaria: Battista Grassi e la scoperta del ciclo di trasmissione della malaria. En: Grassi B. Studi di uno zoologo sulla malaria. Giunti Gruppo Editoriale, Firenze 1998; p 22.

7.- Columela. Los doce libros de agricultura que escribiera en latín Lucio Junio Moderato Columela, traducidos al castellano por D. Juan María Alvarez de Sotomayor y Rubio. Imprenta de D. Miguel de Burgos, Madrid 1824; I (5): 22.
8.- Publio Virgilio Marón. Geórgicas, libro III. En: La Eneida, Bucólicas y Geórgicas. Editorial Iberia S.A., Barcelona 1959; III: 385.

9.- Vitruvio Polión M. Los diez libros de arquitectura. Traducidos del latín i comentados por D. Joseph Ortiz i Sanz. De Orden Superior en la Imprenta Real, Madrid 1787; 1 (IV): 15 .

10.- Rubner M. Tratado de Hijiene, tomo I, pág.12, en: Tagle Rodríguez. El alcantarillado de las casas. Imprenta y Litografía Universo, Santiago de Chile 1908; p 4.

11.- Bechmann G. Salubrité urbaine. Distribution d'eau assainessement. Baudry et Cie., Libraires-Éditeurs. Paris 1888; pp 34-9.

12.- Shoe L T. Vitruvius. En: Collier's Encyclopedia. Crowell Collier and MacMillan, Inc., USA 1967; 23: 179.

13.- Leonardo Da Vinci. Figuras humanas. En: Cuadernos. Edición de H. Anna Suh. Parragon Books Ltd. Bath, RU 2006; II: 42-3.

14.- Clini P, Taus P. Da fano a Fanum Fortunae passeggiando tra memorie e segni della cità vitruviana. www.earcom.org/ Atti2004/clini.pdf

15.- Taus P. Virtual reality in the study for archeological sites: a data-base for Fanum Fortunae. International Archives of the Photogrammatry, Remote sensing and spatial information sciences 2003; XXXIV (5/w12): 330-4. 